\title{
Observation for the High-Speed Oblique Collision of Metals
}

\author{
Akihisa Mori ${ }^{1, a}{ }^{*}$, Shigeru Tanaka ${ }^{2, b}$ and Kazuyuki Hokamoto ${ }^{3, c}$ \\ ${ }^{1}$ Faculty of Engineering, Sojo University, Ikeda 4-22-1 Kumamoto, 860-0082 Japan \\ 2 Institute of Pulsed Power Science, Kumamoto University, 2-39-1 Kurokami, Chuo-ku, \\ Kumamoto, 860-8555 Japan \\ amakihisa@mec.sojo-u.ac.jp, ${ }^{\mathrm{b}}$ tanaka@mech.kumamoto-u.ac.jp, \\ chokamoto@mech.kumamoto-u.ac.jp
}

Keywords: Oblique Collision, Explosive Welding, Interfacial Waves, Metal Jet, Numerical Simulation

\begin{abstract}
In explosive welding, it is known well that the collision angle and collision velocity are the important parameters to achieve good welding. In addition, generations of a metal jet and the interfacial waves are important for the explosive welding conditions. To know the parameters and the collision conditions, the optical observation and the numerical simulation for the oblique collision using a powder gun were done by the authors. A metal jet was observed clearly by using a powder gun and wavy interface was generated without the intermetallic layer for the reactive materials by controlling the welding conditions. In this investigation, the results of the optical observations and the numerical analysis for similar and dissimilar material combinations were reported.
\end{abstract}

\section{Introduction}

Explosive welding technique is known well as the welding method to weld strongly for the two metal plates of similar and/or dissimilar material combinations. In explosive welding technique, a metal flyer plate is accelerated by the detonation of explosive and is collided to another metal plate (base plate) with a certain angle at high velocity. A good welding is achieved with generating the interfacial waves in the welded interface and the metal jet at the collision point when the velocity and the angle collided are within the suitable range [1, 2]. Therefore, to achieve the optimal welding conditions for the difficult-to-weld materials, it is necessary to know the parameters and the collision phenomena, such as the metal jet generations and the interfacial waves. The mechanism of interfacial waves and the metal jet generation have been studied theoretically and/or numerically by many researchers [3-5]. Onzawa et al. [6] reported about the characteristics of metal jet generated by the collision of similar and dissimilar metals set on parallel and angular arrangement using a high-speed streak camera. The observation for the metal jet generation is difficult by the optical observation system because the detonation gas spreads out rapidly with the high velocity which is faster than the flying velocity of metal. From the weldability window proposed by Wittman [7] and Deribas [8], claddings same as explosive welding can be obtained when a metal plate collides obliquely at high velocity. To know the inclined collision, same as the phenomena of explosive welding, a powder gun was applied to observe the high-speed oblique collision, which is same as the phenomena of explosive welding, without the influence of detonation gas. And the numerical simulation using SPH solver in ANSYS AUTODYN software was used to understand the material behavior in the high-speed oblique collision, comparing with the experimental results. 


\section{Experimental Procedure}

Experimental setup to observe the high-speed oblique collision is shown in Figure 1. A powder gun set on Institute of Pulsed Power Science in Kumamoto University was used to accelerate the metal plate. A pure copper and magnesium alloy AZ31, which diameter were $32 \mathrm{~mm}$ and thickness was $3 \mathrm{~mm}$ or $5 \mathrm{~mm}$, were applied as the flyer and target plate. The flyer plate was combined the sabot made by Ultra high molecular weight polyethylene (UHPE) as the projectile. The projectile was set in the barrel of the powder chamber side. The copper weight-control plate was placed behind the flyer plate to control the flying velocity of projectile. The target plate put into a PMMA target holder was arranged on the target stand with an inclined angle $(\theta=7,10$, $15,20)$ in the target chamber. For the optical observation of the oblique collision, High-speed video camera (HPV-1, Shimadzu corp., capable of recording up to 1 million fps) was placed at the side of the target chamber and was located in the opposite side of the light across the target chamber. Smokeless and the black gunpowder were set in the powder chamber. After the target chamber was in a vacuum, the black gunpowder was ignited.

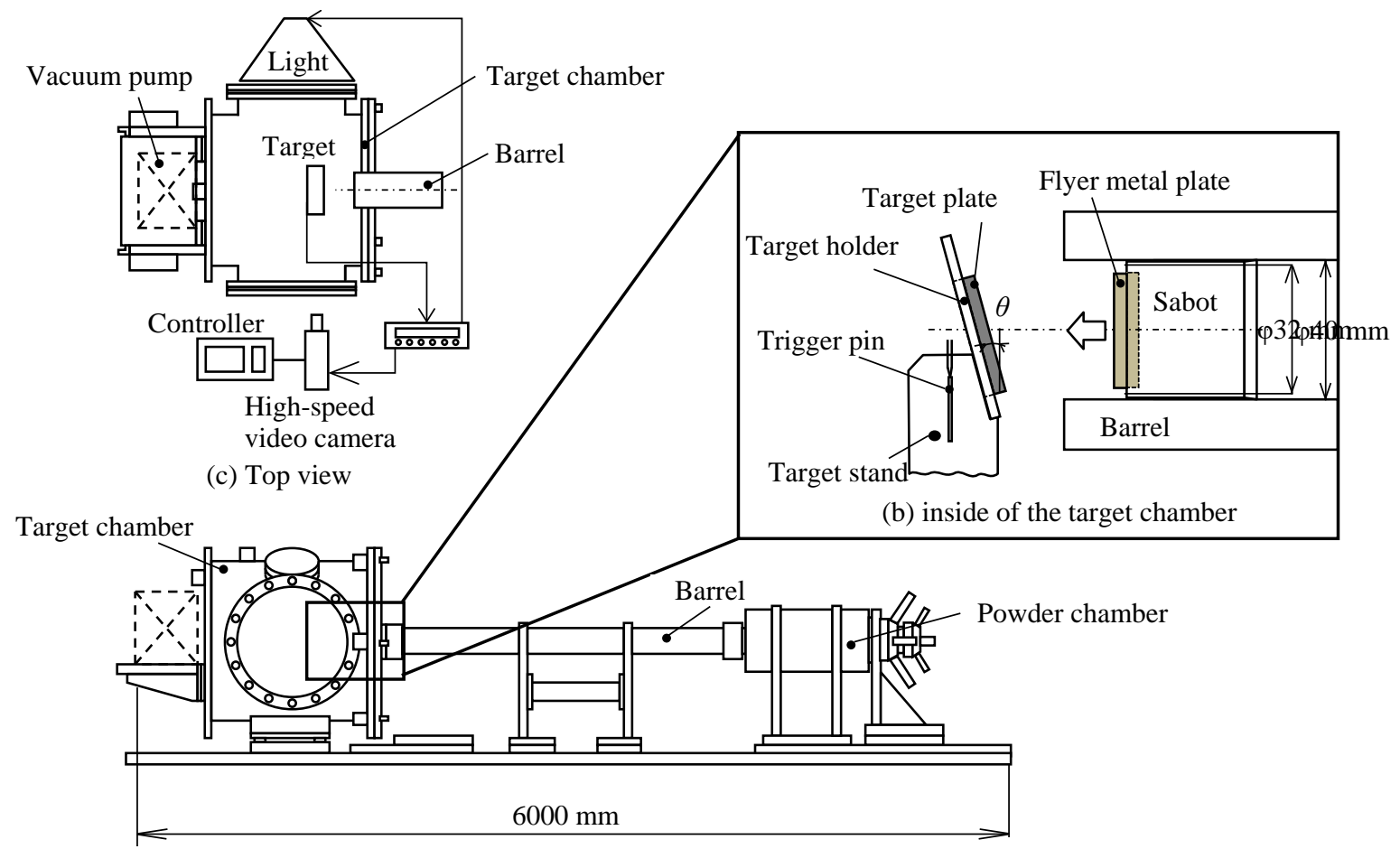

(a) Whole view of a powder gun used

Fig. 1 Schematics of experimental setup used

\section{Numerical Analysis}

Explicit dynamics software ANSYS AUTODYN was used and the 2-dimensional planer symmetry was applied to know the detail of the oblique collision at high velocity numerically. A target plate and a projectile were modeled by two solvers, which were the Smoothed Particle Hydrodynamics (SPH) solver and the Lagrangian solver. The $60 \%$ thickness part on the collision side of the metal plate was modeled by the SPH solver, and the remaining $40 \%$ was applied by the Lagrangian. The particle size of SPH solver and the mesh size of Lagragian solver were fixed at $0.05 \mathrm{~mm}$ and $0.03 \mathrm{~mm}$ when the thickness of metal plates was $5 \mathrm{~mm}$ and $3 \mathrm{~mm}$ respectively. The Mie-Grüneisen form shock equation of stat and the Johnson-Cook strength model were applied for each material. The material parameters for each equation are referred from the reports $[9,10]$. 


\section{Results and discussion}

Examples of framing photographs after the starting of collision were shown in Figure 2. The metal jets generation for the similar and dissimilar material combinations could be observed clearly in the oblique collision, which was the same condition in the explosive welding process, when a powder gun was used. The metal jets were generally dark and not bright when the copper and copper oblique collision. In the case of using a magnesium alloy regardless of the similar / dissimilar combination, bright metal jets were observed shown in Figure 2 (b) and (c). In addition, it was confirmed that two types of metal jet, the central jet and the surrounding jet, were generated when the installation angle of the plate was 15 degrees or more, as shown in Figure 2 (a) and (c).

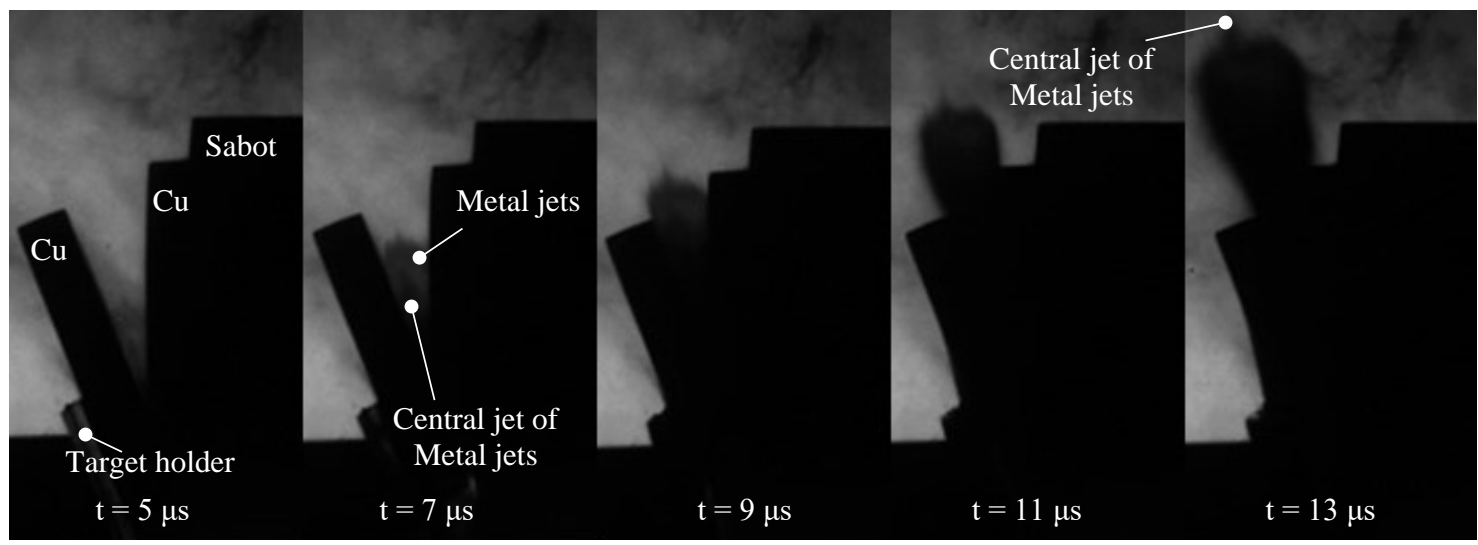

(a) metal jets of similar metals (copper/copper): $V_{p}=630 \mathrm{~m} / \mathrm{s}, \theta=20^{\circ}$

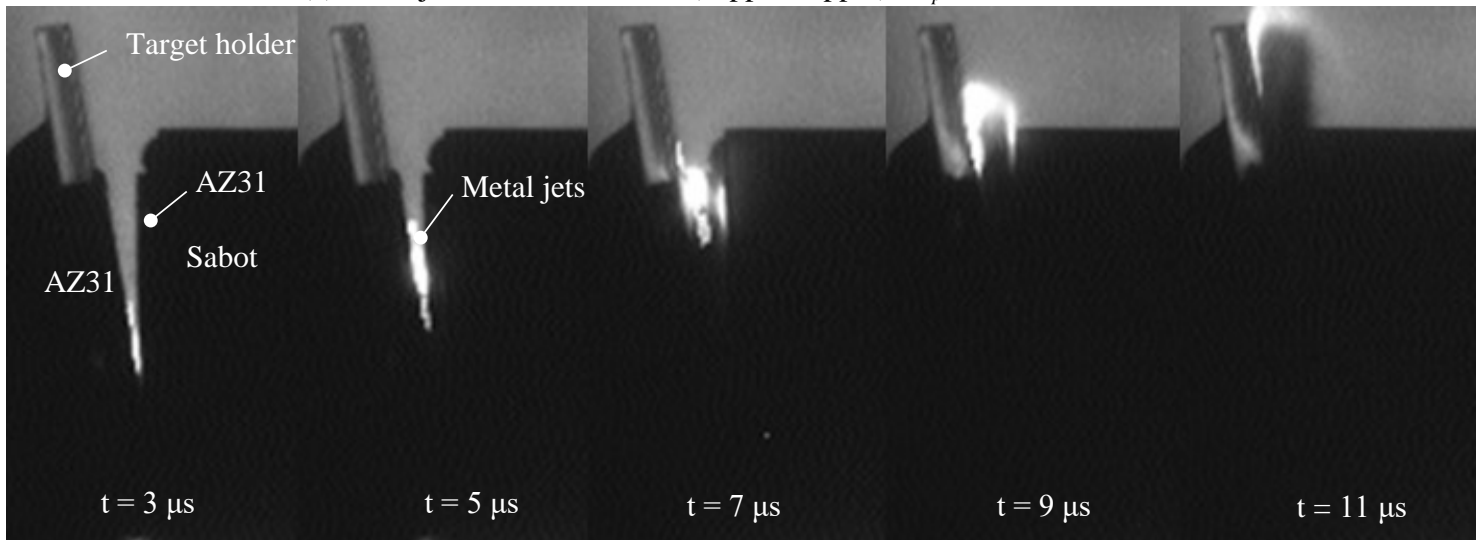

(b) metal jets of similar metals (AZ31/AZ31): $V_{p}=420 \mathrm{~m} / \mathrm{s}, \theta=7^{\circ}$

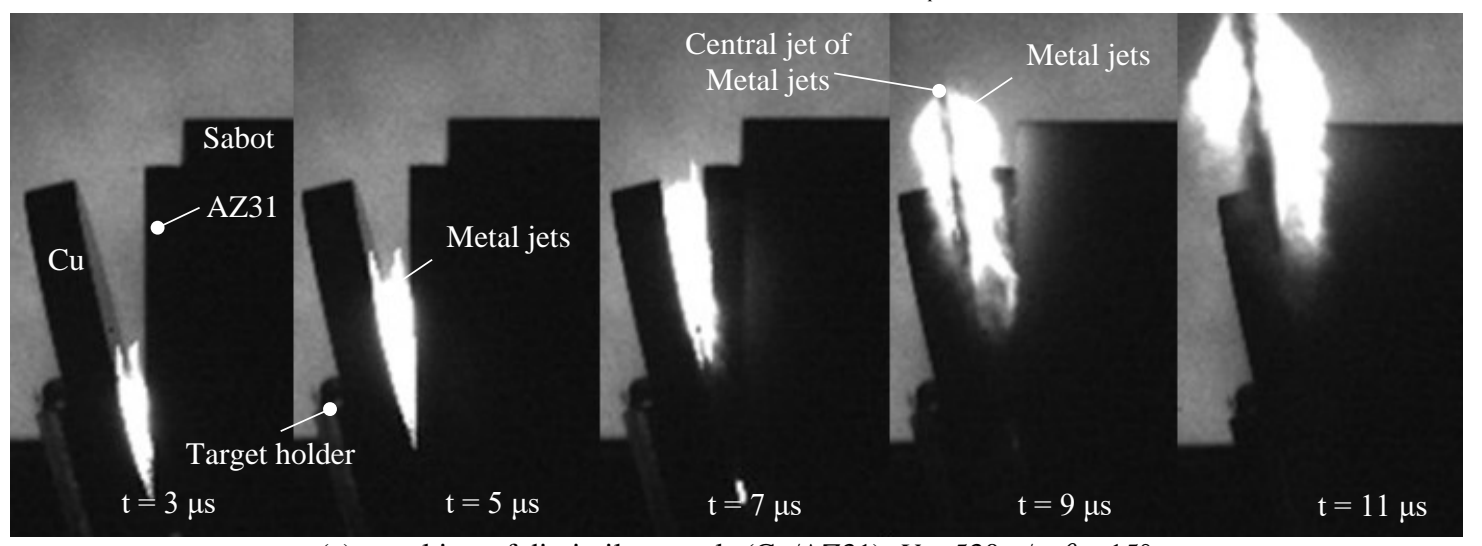

(c) metal jets of dissimilar metals (Cu/AZ31): $V_{p}=530 \mathrm{~m} / \mathrm{s}, \theta=15^{\circ}$

Fig. 2 Metal jet generations in the oblique collsino of similar and dissimilar material combition 
From the formula of the relation for the collision velocity, the collision point velocity and the collision angle [1], the collision point velocity was 1810, 3440 and $1780 \mathrm{~m} / \mathrm{s}$ in the condition of Figure 2(a), (b) (c) respectively. And the moving velocity of the top of metal jets obtained from these photographs was 3540, 5350 and $3560 \mathrm{~m} / \mathrm{s}$, which was about twice the collision point velocity.

Numerical results for the oblique collision of $\mathrm{Cu} / \mathrm{Cu}$, AZ31/AZ31 and $\mathrm{Cu} / \mathrm{AZ31}$ using ANSYS AUTODYN-2D were shown in Figure 3. The particle velocity constituting the metal jets agreed with the experimental results. In the case of Figure 3(a), the large-size interfacial waves which height was about $1 \mathrm{~mm}$ were formed from the center to the end of metals numerically although the small interfacial waves were formed around the starting of the collision. On the other hand, in the case of magnesium alloys, even the similar material combination, small interfacial waves were simulated from the starting of the collision to end. In the temperature contour of the numerical results, the metal jets were increased at $2000 \sim 3000 \mathrm{~K}$ in the case of copper and copper metals and increase over $5000 \mathrm{~K}$ in the magnesium alloy. Since the melting point of the magnesium alloy is $650{ }^{\circ} \mathrm{C}$ and the boiling point is $1090{ }^{\circ} \mathrm{C}$, it was thought that magnesium jets were vaporized instantaneously, considering only the temperature state. However, it was unclear what the phase of metal jets specifically was because of the highpressure condition at the time of collision. A slight difference in the state of temperature and pressure has been speculated that two types of jet could be observed in experimental results. From the numerical analysis, the metal jets were composed only of magnesium alloy in the case of dissimilar oblique collision. It was possible that the jets were composed mainly of the lowdensity material.

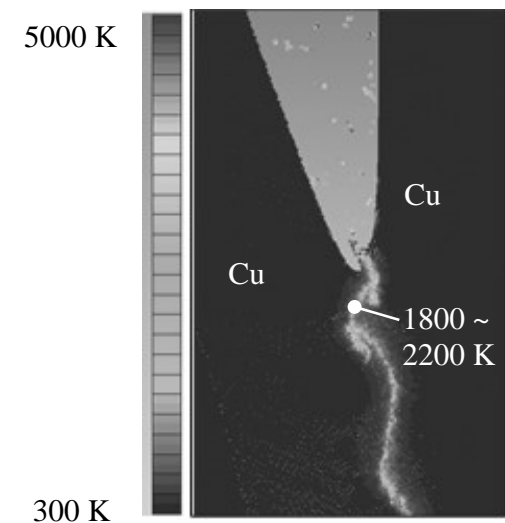

(a) $\mathrm{Cu} / \mathrm{Cu}$, $V_{p}=630 \mathrm{~m} / \mathrm{s}, \theta=20^{\circ}$

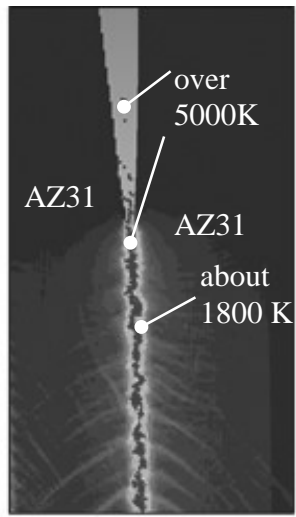

(b) AZ31/AZ31, $V_{p}=420 \mathrm{~m} / \mathrm{s}, \theta=7^{\circ}$

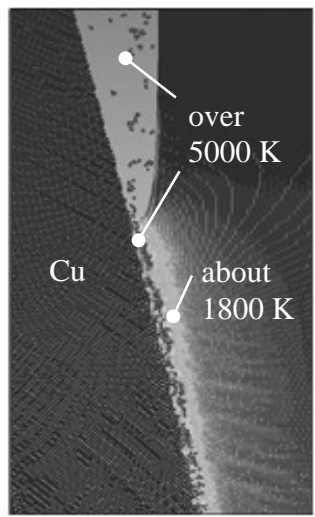

(c) $\mathrm{Cu} / \mathrm{AZ31}$, $V_{p}=530 \mathrm{~m} / \mathrm{s}, \theta=15^{\circ}$

Fig.3 Temperature contour of the collided interface obtained from the numerical simulation

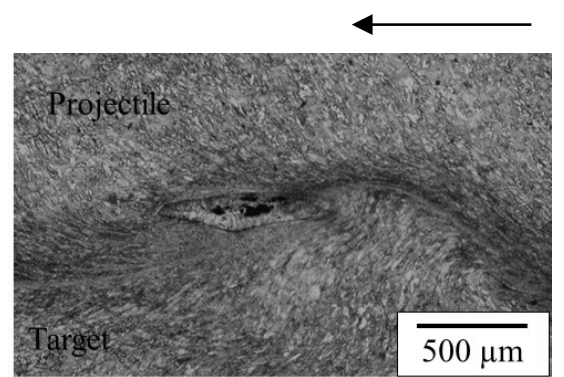

(a) $\mathrm{Cu} / \mathrm{Cu}$ in $V_{p}=630 \mathrm{~m} / \mathrm{s}, \theta=20^{\circ}$
Welding direction

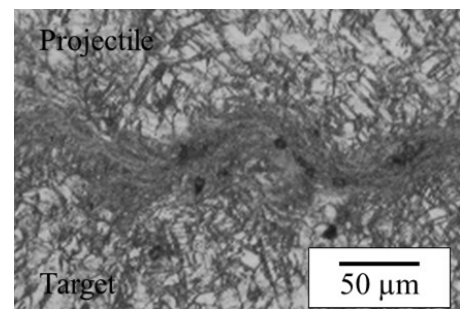

(b) AZ31/AZ31 in $V_{p}=420 \mathrm{~m} / \mathrm{s}, \theta=7^{\circ}$

Fig.4 Welded interface of the recovered sample 
Figure 4 shows a welding cross-section of recovered in experimental samples. In the similar metal combination, a good welding was achieved with forming the interfacial waves in the same as the explosive welding. However, a good welding was not achieved in the dissimilar metal combination. In the case of magnesium alloys, it could not be achieved to weld at high velocity or large collision angle. In the case of the $\mathrm{Cu} / \mathrm{Cu}$ oblique collision, as shown in Figure 4(a), molten parts were observed in the welded interface. Therefore, it was thought that the separation or the destruction was occurred at the molten parts when a magnesium alloy was used.

\section{Summary}

The phenomena of high-speed oblique collision of the two metals were investigated in the experiments using the optical observation system and in the numerical simulation. In the optical observations metal jets were observed clearly and two types of metal jets were observed when the setup angle was 15 degrees and more. And it was confirmed that the top of metal jets was propagated at approximately twice of the collision point velocity. In the numerical results, it could be confirmed the behaviors and temperature conditions of the collided interface and the metal jets, which were difficult to measure by the experimental method.

\section{References}

[1] B. Crossland, Explosive Welding of Metals and its Application, Oxford University Press, 1982.

[2] M. A. Meyers, et al., Dynamic behavior of materials, John Wiley \& Sons, 1994.

[3] C. Chemin, T. Qingming, Mechanism of Wave Formation at the Interface in Explosive Welding, Acta Mechanica Sinica, 5-2 (1989), 97-108. https://doi.org/10.1007/bf02489134

[4] S. Kakizaki, M. Watanabe and S. Kumai, Simulation and Experimental Analysis of Metal Jet Emission and Weld Interface Morphology in Impact Welding, Materials Trans., 52-5 (2011), 1003-1008. https://doi.org/10.2320/matertrans.l-mz201128

[5] G.R. Cowan, A.H. Holtman, Flow Configurations in Colliding Plates: Explosive Bonding, J. Appl. Phys., 34-4 (1963), 928-939. https://doi.org/10.1063/1.1729565

[6] T. Onzawa, Y. Ishii, Fundamental Studies on Explosive Welding, Trans. Japan Welding Society, 6-2 (1975), 98-104.

[7] R.H. Wittman, The Influence of Collision Parameters on the Strength and Microstructure of an Explosion Welded Aluminum Alloy, Proc. 2nd Sym. on Use of Explosive Energy in Manufacturing Metallic Materials of New Properties and Possibilities of Application thereof in the Chemical Industry, (1973), 153-168.

[8] A.A. Deribas, V.A. Simonov, and I.D. Zakcharenko, Investigation of Explosive Welding Parameters for Arbitrary Combinations of Metals and Alloys, Proc. $5^{\text {th }}$ Int. Conf. on High Energy Rate Fabrication, (1975),4.1.1-4.1.24.

[9] Ulacia, I., Salisbury, C. P., Hurtado, I., Worswick, M. J., Tensile characterization and constitutive modeling of AZ31B magnesium alloy sheet over wide range of strain rates and temperatures, J. Mater. Process. Technol., 211(5), 830-839 (2011). https://doi.org/10.1016/j.jmatprotec.2010.09.010

[10] A. Mori, S. Tanaka, and K. Hokamoto, Optical observation of metal jet generated by high speed inclined collision, Proc. SPIE 10328, (2017), 103281Q.

https://doi.org/10.1117/12.2270473 